\title{
Real-World Study of Once-Daily, Extended-Release Tacrolimus Versus Twice-Daily, Immediate-Release Tacrolimus in Kidney Transplantation: Clinical Outcomes and Healthcare Resource Utilization
}

\author{
Bing Ho $\cdot$ Hardik Bhagat $\cdot$ Jason J. Schwartz $\cdot$ Kofi Atiemo \\ Amna Daud $\cdot$ Raymond Kang $\cdot$ Samantha E. Montag · \\ Lihui Zhao $\cdot$ Edward Lee $\cdot$ Anton I. Skaro $\cdot$ Daniela P. Ladner
}

Received: August 23, 2018 / Published online: April 2, 2019

(C) The Author(s) 2019

\section{ABSTRACT}

Introduction: Real-world data with extendedrelease tacrolimus (ER-T) are lacking in the USA. This study examined clinical outcomes and healthcare resource utilization in kidney transplant patients receiving ER-T in clinical practice.

Methods: This was a retrospective, single-center analysis (February-June 2016) using data from

Enhanced Digital Features To view enhanced digital features for this article go to https://doi.org/10.6084/ m9.figshare.7687397.

B. Ho $\cdot$ K. Atiemo $\cdot$ A. Daud · R. Kang

S. E. Montag · L. Zhao · D. P. Ladner $(\bowtie)$

Northwestern University Transplant Outcomes Research Collaborative (NUTORC), Comprehensive Transplant Center, Northwestern University, $676 \mathrm{~N}$. St. Clair Street, 19th Floor, Chicago, IL 60611, USA e-mail: dladner@nm.org

H. Bhagat · J. J. Schwartz · E. Lee

Medical Affairs, Americas, Astellas Pharma Global Development, Inc., 1 Astellas Way, Northbrook, IL 60062, USA

S. E. Montag · L. Zhao

Department of Preventive Medicine, Northwestern University, 680 N. Lake Shore Drive, Suite 1400, Chicago, IL 60611, USA

\section{A. I. Skaro}

Department of Surgery, Schulich School of Medicine and Dentistry, Western University, St. Joseph's Hospital, 268 Grosvenor Street, Rm. E3-117, London, ON N6A 4V2, Canada
Northwestern University's Enterprise Data Warehouse. Adult patients receiving a kidney transplant in the preceding 4 years, treated de novo or converted to ER-T from immediaterelease tacrolimus (IR-T) within 10 days posttransplantation, and maintained on ER-T (at least 3 months) were included. Patients were matched for demographic and clinical characteristics with IR-T-treated control patients. Endpoints included clinical outcomes and healthcare resource utilization up to 1 year posttransplantation.

Results: A total of 19 ER-T-treated patients were matched with 55 IR-T-treated patients. No ER-T-treated patients experienced biopsy-confirmed acute rejection (BCAR) or graft failure versus 3 (5.5\%) and 3 (5.5\%) IR-T-treated patients, respectively. Mean estimated glomerular filtration rate (eGFR), the number of all-cause outpatient visits, readmissions, and all-cause hospitalization days were comparable between groups. Tacrolimus trough levels, days to target level (6-10 ng/mL), and number of required dose adjustments were also similar.

Conclusion: Real-world clinical outcomes and healthcare resource utilization were similar with ER-T and IR-T. Larger studies will need to investigate the trend toward fewer BCAR events, and increased graft survival with ER-T.

Funding: Astellas Pharma Global Development, Inc.

Plain Language Summary: Plain language summary available for this article. 
Keywords: Calcineurin inhibitor; Glomerular filtration rate (GFR); Graft survival; Immunosuppressant; Kidney (allograft) function/dysfunction; Patient characteristics; Tacrolimus; Urology

\section{PLAIN LANGUAGE SUMMARY}

Immunosuppressive drugs, such as tacrolimus, are crucial in patients after an organ transplant. Without these drugs, the risk of the body rejecting the new organ is increased, which can leave the patient in a life-threatening condition. Tacrolimus was originally available as a twicedaily, immediate-release formulation (IR-T), with rapid absorption of the drug into the body soon after oral administration. A once-daily, extended-release formulation (ER-T) was subsequently developed to reduce the risk of underand overexposure to tacrolimus by releasing the drug more slowly over a prolonged period of time. There is a lack of data available from clinical practice in the USA regarding the use of ER-T in kidney transplantation. This study compared clinical outcomes and use of healthcare resources over 1 year in kidney transplant patients receiving IR-T or ER-T at a single US transplant center. There was no statistically significant difference in the incidence of kidney rejection or failure in patients receiving ER-T compared with IR-T, although there was a trend toward a lower incidence with ER-T. There was also no statistical difference between the two groups in the use of healthcare resources; however, there was a trend toward fewer days spent hospitalized during the first year, and fewer outpatient clinic visits during the first 3 months after transplantation with ER-T. This study shows that the use of ER-T in US clinical practice can achieve promising outcomes. However, more studies are now needed to investigate whether prescribing ER-T could lead to increased kidney survival and lower healthcare costs compared with IR-T.

\section{INTRODUCTION}

Extended-release tacrolimus (ER-T) is an oral, once-daily, immunosuppressive agent that was developed to simplify dosing regimens while retaining the safety and efficacy profile of the immediate-release formulation. In 2013, the US regulatory authority granted approval for ER-T on the basis of data from two large randomized controlled trials (RCTs), conducted in 638 and 667 de novo kidney transplant recipients, respectively $[1,2]$. ER-T has, however, been available in the European Union since 2007.

Compared with twice-daily, immediaterelease tacrolimus (IR-T), the extended-release formulation has been shown to decrease intrapatient variability in tacrolimus exposure [3, 4] and offer a smoother blood concentration-time profile [3]. ER-T may therefore provide a more consistent area under the concentration-time curve over the long term versus the immediaterelease formulation. Additionally, once-daily dosing with ER-T has been shown to improve medication adherence compared with IR-T in both kidney and liver transplantation [5-7]. As both high intrapatient variability in tacrolimus exposure and medication nonadherence are associated with reduced graft survival and donor-specific antibody development [8-13], targeting both risk factors with a formulation of tacrolimus that potentially addresses these concerns could be a relevant mechanism to improve long-term outcomes.

In the USA, the overall 5-year kidney graft survival rate ranges from $81 \%$ to approximately $90 \%$, depending on race [14], while the 10 -year graft survival rate is between approximately $35 \%$ and 50\% [15]. Although 1-year kidney graft survival rates have increased in recent years (currently greater than 90\%) [14, 15], long-term graft survival in the USA remains 25\% lower than in other countries with well-developed kidney transplant systems [16].

Therefore, there is an urgent need to improve long-term outcomes, a goal that could be accomplished by optimizing immunosuppressive regimens. Tacrolimus has a narrow therapeutic index [17], and so optimizing its exposure is essential, especially as antibodymediated rejection, now linked to inadequate immunosuppression, is increasingly recognized as a major cause of late graft failure $[13,18]$.

The use of ER-T is low in clinical practice in the USA due to higher clinician familiarity with 
IR-T formulations and limited perceived differences in efficacy and safety compared with IR-T formulations. Notably, the pivotal phase III RCTs for ER-T conducted in de novo kidney transplant recipients excluded patients receiving induction with lymphocyte-depleting agents, an induction strategy employed by many US transplant centers today. Real-world database studies can thus provide further insights into treatment patterns and outcomes for a more heterogeneous patient population versus those enrolled in larger, controlled registrational studies. To our knowledge, no studies have been undertaken to date that examine the clinical outcomes and healthcare resource utilization of kidney transplant patients treated with ER-T under real-world conditions in the modern era of immunosuppression. As such, we utilized data from the Northwestern Medicine Enterprise Data Warehouse with the aim of conducting a retrospective, real-world study of clinical outcomes and healthcare resource utilization in kidney transplant patients receiving ER-T versus IR-T at a single US transplant center.

\section{METHODS}

\section{Study Design}

This was a retrospective, single-center, database analysis conducted between February and June 2016. Data for eligible adult patients who had received a kidney transplant during the 4 years preceding the study (January 2012-January 2016) were extracted from the Northwestern Medicine Enterprise Data Warehouse. This database is a single, comprehensive, integrated repository that captures all electronic health records at clinical facilities within the Northwestern Medicine integrated healthcare system (Illinois, USA). Extracted data were de-identified before analysis. This retrospective database analysis study had approval of the Northwestern University Institutional Review Board (IRB\#STU0020221) and adhered to the 1964 Helsinki declaration and its later amendments or comparable ethical standards. As a retrospective study, informed consent of individuals was not required.

\section{Study Population}

Data were collected for adult patients who had received a kidney transplant at Northwestern Medicine within the study period, who were initiated or switched to routine maintenance immunosuppression with once-daily, ER-T (Astagraf XL ${ }^{\circledR}$, Astellas Pharma US, Inc., Northbrook, IL; also known outside the USA as Advagraf ${ }^{\circledR}$, Graceptor ${ }^{\circledR}$, and Prograf ${ }^{\circledR}$ XL) within 10 days of transplantation, and who continued this regimen for at least 3 months. Per center protocol for tacrolimus initiation, patients received IR-T if therapy was initiated during the initial hospital stay and, subsequently, were switched to ER-T upon hospital discharge. If the first dose of tacrolimus therapy was initiated after hospital discharge, patients were started and maintained on ER-T. Patient cases were matched with control patients who were taking either generic or branded forms of IR-T. Those receiving maintenance therapy with drugs other than tacrolimus (e.g., cyclosporine, belatacept, or sirolimus), or who were participating in a clinical trial during the study period, were excluded.

\section{Endpoints}

Clinical outcomes and healthcare resource utilization were assessed at 30, 90, and 365 days post-transplantation. Clinical outcomes included the incidence of biopsy-confirmed acute rejection (BCAR), graft loss (defined as a return to dialysis, primary graft nonfunction, or dialysis for more than 3 months), the need for retransplantation, and mortality. Estimated glomerular filtration rate (eGFR) by the Modification of Diet in Renal Disease- 4 formula, and the proportion of patients experiencing delayed graft function (defined as a requirement for renal replacement therapy within 7 days of transplantation) were also analyzed. Healthcare resource utilization was tracked and included the number of all-cause outpatient clinic visits, number of all-cause readmissions, total number of all-cause hospitalization days, and total number of all-cause emergency room visits to Northwestern University facilities. The number 
of renal biopsies within 365 days of transplantation was also assessed.

The time to reach a tacrolimus trough level of $6-10 \mathrm{ng} / \mathrm{mL}$ (therapeutic target) was evaluated, as well as the number of dose adjustments required to reach this target. Tacrolimus trough levels were also compared between patients treated with the IR-T formulation (using trough levels from at least 30 days post-transplantation), and those who received ER-T (from at least 30 days after initiation or switching from the IR-T to the ER-T formulation).

\section{Data Analysis and Statistical Methods}

Patients receiving ER-T were matched 1:3 with those receiving IR-T. Patients were propensity score matched for 15 patient demographic and clinical characteristics present at the time of their first transplant procedure. These characteristics included sex, race/ethnicity, payer type, organ donor type (living versus deceased), induction regimen, steroid use, panel reactive antibody score, kidney donor profile index, transplantation status (retransplant), time on dialysis, comorbid conditions (such as obesity, diabetes, hypertension, or focal segmental glomerulosclerosis), number of human leukocyte antigen mismatches, and number of days post-transplantation prior to tacrolimus administration. Among the possible control patients who met these criteria for a given case, the three with the closest propensity score to that of the case were selected. This process was conducted in a randomly assigned order by case; selection of controls was made without replacement.

Recipient age and the Charlson Comorbidity Index were excluded from the propensity score model and exact-matched instead. Specifically, an exact match was performed on tertiles of age and Charlson Comorbidity Index, and quintiles of propensity score, in order to limit selection bias.

For the analysis of eGFR, values greater than $60 \mathrm{~mL} / \mathrm{min} / 1.73 \mathrm{~m}^{2}$ were truncated, with values reported only as high as $60 \mathrm{~mL} / \mathrm{min} / 1.73 \mathrm{~m}^{2}$ given that values above this threshold can be unreliable [19].
The comparisons between groups were made using generalized estimating equations to account for the match. Comparisons were considered statistically significant at the $p<0.05$ level. All analyses were performed using SAS ${ }^{\circledR}$ version 9.4, and $R$ version 3.2.2 using the $R$ Studio interface.

\section{RESULTS}

\section{Patient Demographics and Clinical Characteristics}

During the observation period, 19 patients were initiated on treatment with ER-T, or were converted to treatment from IR-T to ER-T within 10 days post-transplantation, and continued therapy for a period of at least 3 months. Of these 19 patients, $17(89.5 \%)$ received ER-T as part of their planned discharge immunosuppression regimen, one $(5.3 \%)$ received ER-T because of loss of insurance, and one $(5.3 \%)$ received ER-T because of concern over deteriorating renal function (no further details available). The 19 patients were subsequently matched with 55 patients treated with IR-T (Table 1). The exact proportions of patients receiving generic versus branded forms of IR-T were unavailable; however, use of generic preparations increased during the study period.

During the study period, four patients (21.1\%) switched from ER-T to IR-T. One of these patients showed borderline changes on a protocol biopsy at 3 months, with new onset BK viruria; one had changes suggestive of borderline cellular infiltrates with subtherapeutic tacrolimus levels at 3 months; and one patient was highly sensitized and experienced supratherapeutic tacrolimus levels on the extended-release preparation. The fourth patient switched to IR-T because of cost differences between generic IR-T and the branded ER-T formulation.

As anticipated, there were no significant differences in baseline covariates between the ER-T and IR-T groups after propensity score and exact matching (Table 1). Most patients receiving ER-T were male $(63.2 \%)$, with a mean \pm standard deviation (SD) age of $49.5 \pm 16.3$ years (range 
Table 1 Patient demographics and clinical characteristics

\begin{tabular}{|c|c|c|c|}
\hline Parameter & $\begin{array}{l}\text { Extended-release tacrolimus } \\
(N=19)\end{array}$ & $\begin{array}{l}\text { Immediate-release tacrolimus } \\
(N=55)\end{array}$ & $p$ value \\
\hline Sex & & & 0.90 \\
\hline Female & $7(36.8)$ & $21(38.2)$ & \\
\hline Male & $12(63.2)$ & $34(61.8)$ & \\
\hline Race/ethnicity & & & 0.45 \\
\hline Asian & $2(10.5)$ & $5(9.1)$ & \\
\hline Hispanic & $6(31.6)$ & $15(27.3)$ & \\
\hline Non-Hispanic black & $1(5.3)$ & $14(25.5)$ & \\
\hline Non-Hispanic white & $10(52.6)$ & $21(38.2)$ & \\
\hline Insurance & & & 0.39 \\
\hline Medicaid & $1(5.3)$ & $6(10.9)$ & \\
\hline Medicare & $7(36.8)$ & $26(47.3)$ & \\
\hline Private & $11(57.9)$ & $23(41.8)$ & \\
\hline Education & & & 0.39 \\
\hline Attended some college & $1(5.3)$ & $18(32.7)$ & \\
\hline $\begin{array}{l}\text { Bachelors/associate } \\
\text { degree }\end{array}$ & $5(26.3)$ & $11(20.0)$ & \\
\hline Grade school & $2(10.5)$ & $4(7.3)$ & \\
\hline High school & $10(52.6)$ & $19(34.5)$ & \\
\hline Postgraduate degree & $1(5.3)$ & $3(5.5)$ & \\
\hline Primary language & & & 0.42 \\
\hline English & $15(78.9)$ & $47(85.5)$ & \\
\hline Spanish & $4(21.1)$ & $6(10.9)$ & \\
\hline Other & $0(0.0)$ & $2(3.6)$ & \\
\hline Obesity & & & 0.19 \\
\hline Normal weight & $5(26.3)$ & $16(29.1)$ & \\
\hline Obese & $6(31.6)$ & $18(32.7)$ & \\
\hline Overweight & $5(26.3)$ & $21(38.2)$ & \\
\hline Underweight & $3(15.8)$ & $0(0.0)$ & \\
\hline PRA & & & 0.68 \\
\hline Highly sensitized $[81+]$ & $2(10.5)$ & $4(7.3)$ & \\
\hline Sensitized $[21-80]$ & $3(15.8)$ & $11(20.0)$ & \\
\hline Unsensitized [0-20] & $14(73.7)$ & $40(72.7)$ & \\
\hline
\end{tabular}


Table 1 continued

\begin{tabular}{|c|c|c|c|}
\hline Parameter & $\begin{array}{l}\text { Extended-release tacrolimus } \\
(N=19)\end{array}$ & $\begin{array}{l}\text { Immediate-release tacrolimus } \\
(N=55)\end{array}$ & $p$ value \\
\hline \multicolumn{3}{|l|}{ Induction regimen } & 0.13 \\
\hline Alemtuzumab & $11(57.9)$ & $47(85.5)$ & \\
\hline Basiliximab & $7(36.8)$ & $8(14.5)$ & \\
\hline Neither & $1(5.3)$ & $0(0.0)$ & \\
\hline \multicolumn{3}{|c|}{ Steroid use after 3 months post-transplantation } & 0.11 \\
\hline Yes & $9(47.4)$ & $17(30.9)$ & \\
\hline No & $10(52.6)$ & $37(67.3)$ & \\
\hline Unknown & $0(0.0)$ & $1(1.8)$ & \\
\hline \multicolumn{3}{|l|}{ Prior transplant } & 0.79 \\
\hline No & $19(100.0)$ & $52(94.5)$ & \\
\hline Yes & $0(0.0)$ & $3(5.5)$ & \\
\hline \multicolumn{3}{|c|}{ Number of HLA-A mismatches } & 0.34 \\
\hline 0 & $5(26.3)$ & $19(34.5)$ & \\
\hline 1 & $12(63.2)$ & $31(56.4)$ & \\
\hline 2 & $2(10.5)$ & $5(9.1)$ & \\
\hline \multicolumn{3}{|c|}{ Number of HLA-B mismatches } & 0.49 \\
\hline 0 & $6(31.6)$ & $13(23.6)$ & \\
\hline 1 & $11(57.9)$ & $41(74.5)$ & \\
\hline 2 & $2(10.5)$ & $1(1.8)$ & \\
\hline \multicolumn{3}{|c|}{ Number of HLA-DR mismatches } & 0.41 \\
\hline 0 & $8(42.1)$ & $20(36.4)$ & \\
\hline 1 & $9(47.4)$ & $29(52.7)$ & \\
\hline 2 & $2(10.5)$ & $6(10.9)$ & \\
\hline \multicolumn{3}{|c|}{ Number of HLA-A, -B, -DR mismatches } & 0.57 \\
\hline 0 & $1(5.3)$ & $1(1.8)$ & \\
\hline 1 & $1(5.3)$ & $1(1.8)$ & \\
\hline 2 & $0(0.0)$ & $1(1.8)$ & \\
\hline 3 & $3(15.8)$ & $7(12.7)$ & \\
\hline 4 & $2(10.5)$ & $11(20.0)$ & \\
\hline 5 & $7(36.8)$ & $18(32.7)$ & \\
\hline 6 & $5(26.3)$ & $16(29.1)$ & \\
\hline
\end{tabular}


Table 1 continued

\begin{tabular}{|c|c|c|c|}
\hline Parameter & $\begin{array}{l}\text { Extended-release tacrolimus } \\
(N=19)\end{array}$ & $\begin{array}{l}\text { Immediate-release tacrolimus } \\
(N=55)\end{array}$ & $p$ value \\
\hline \multicolumn{3}{|l|}{ Diabetes } & 0.94 \\
\hline No & $15(78.9)$ & $44(80.0)$ & \\
\hline Yes & $4(21.1)$ & $11(20.0)$ & \\
\hline \multicolumn{3}{|l|}{ Hypertension } & 0.30 \\
\hline No & $10(52.6)$ & $32(58.2)$ & \\
\hline Yes & $9(47.4)$ & $23(41.8)$ & \\
\hline \multicolumn{3}{|c|}{ Polycystic kidney disease } & 0.63 \\
\hline No & $16(84.2)$ & $48(87.3)$ & \\
\hline Yes & $3(15.8)$ & $7(12.7)$ & \\
\hline \multicolumn{3}{|c|}{ Glomerulonephritis } & 0.62 \\
\hline No & $18(94.7)$ & $51(92.7)$ & \\
\hline Yes & $1(5.3)$ & $4(7.3)$ & \\
\hline \multicolumn{3}{|c|}{ IgA nephropathy } & 0.27 \\
\hline No & $18(94.7)$ & $49(89.1)$ & \\
\hline Yes & $1(5.3)$ & $6(10.9)$ & \\
\hline \multicolumn{3}{|c|}{ Focal segmental glomerulosclerosis } & 0.40 \\
\hline No & $18(94.7)$ & $51(92.7)$ & \\
\hline Yes & $1(5.3)$ & $4(7.3)$ & \\
\hline \multicolumn{3}{|c|}{ Systemic lupus erythematosus } & 0.99 \\
\hline No & $18(94.7)$ & $52(94.5)$ & \\
\hline Yes & $1(5.3)$ & $3(5.5)$ & \\
\hline \multicolumn{3}{|l|}{ Other diagnosis } & 0.99 \\
\hline No & $16(84.2)$ & $49(89.1)$ & \\
\hline Yes & $3(15.8)$ & $6(10.9)$ & \\
\hline
\end{tabular}

$H L A$ human leukocyte antigen, $I g A$ immunoglobulin A, $P R A$ panel reactive antibody

Data are $n(\%)$

23.0-74.0 years) at transplantation. Patients taking ER-T had received dialysis for a mean \pm SD of $3.1 \pm 3.7$ years at the time of transplantation, had a mean kidney donor profile index of $38.1 \pm 23.3 \%$, and a mean Charlson Comorbidity Index score of $3.7 \pm 1.4$ points. Most patients received induction therapy with alemtuzumab
(57.9\%) or basiliximab (36.8\%). The median time between transplantation and initiation of ER-T treatment was 1.5 days (interquartile range 1.3-3.0 days), with patients receiving ER-T for a median of 490.0 days (interquartile range 111.0-632.0 days). 


\section{Mortality and Graft Loss}

No patients (0/19) in the ER-T group experienced a BCAR event compared with 3/55 (5.5\%) patients in the IR-T group. As previously noted, one patient on ER-T showed borderline changes on a protocol biopsy at 3 months with new onset BK viruria and one patient had changes suggestive of borderline cellular infiltrates at 3 months with subtherapeutic tacrolimus levels. Neither of these patients met Banff diagnostic criteria for BCAR. Graft failure did not occur in any patients in the ER-T group (0/19) compared with $3 / 55(5.5 \%)$ patients in the IR-T group. No patients required retransplantation or died during the study, in either cohort.

\section{Renal Function}

No patients in either treatment group experienced delayed graft function post-transplantation. Mean eGFR ranged between 46.2 and $52.6 \mathrm{~mL} / \mathrm{min} / 1.73 \mathrm{~m}^{2}$ across time points and treatment groups. The values were similar in both treatment groups at 30,90, and 365 days post-transplantation $(p=0.71, p=0.51$, and $p=0.47$, respectively), the caveat being that data were capped when patients achieved the maximum eGFR of $60 \mathrm{~mL} / \mathrm{min} / 1.73 \mathrm{~m}^{2}$ (Fig. 1).

\section{Tacrolimus Dose and Trough Levels}

There were no significant differences between the ER-T and IR-T groups with regard to the number of days (mean \pm SD) taken to reach the target trough level of $6-10 \mathrm{ng} / \mathrm{mL}(9.2 \pm 8.3$ versus $7.8 \pm 7.9$ days, respectively; $p=0.18$ ), or the number of dose adjustments required to reach the target trough level $(1.3 \pm 1.3$ versus $0.9 \pm 1.3$ adjustments; $p=0.13$ ). The number of tacrolimus trough levels drawn was similar in both treatment groups at 30, 90, and 365 days post-transplantation (Table 2 ). There was also no significant difference in tacrolimus trough levels (mean \pm SD) beyond 30 days posttransplant with ER-T compared with IR-T $(8.5 \pm 1.2$ versus $7.7 \pm 1.3 \mathrm{ng} / \mathrm{mL}$, respectively; $p=0.51$ ) (Table 3). The dose-unadjusted SDs of tacrolimus trough concentrations were also similar in both treatment groups after 30 days post-transplant.

\section{Clinic Visits and Hospitalization}

The mean number of all-cause outpatient clinic visits by 365 days post-transplantation was 4.8 in the ER-T group and 4.4 in the IR-T group $(p=0.72)$. The mean number of all-cause outpatient clinic visits within 30 days was lower

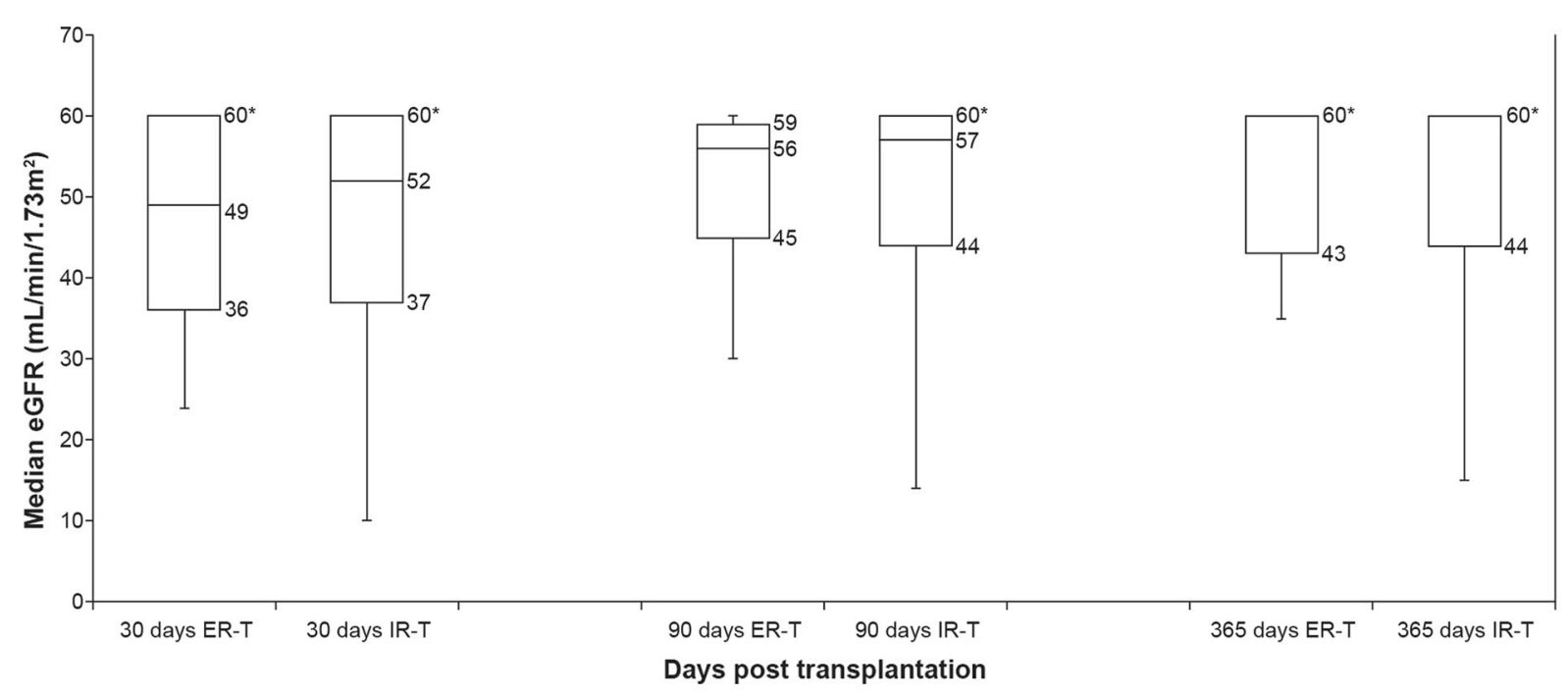

Fig. 1 Median estimated glomerular filtration rate at 30, 90 , and 365 days post-transplantation for patients who received extended-release $(N=19)$ or immediate-release tacrolimus $(N=55)$. Whiskers represent range. ${ }^{*} \mathrm{eGFR}$ was capped at $60 \mathrm{~mL} / \mathrm{min} / 1.73 \mathrm{~m}^{2}$ 
Table 2 Number of tacrolimus trough concentration measurements

\begin{tabular}{lllll}
\hline & $\begin{array}{l}\text { Time point post- } \\
\text { transplant (days) }\end{array}$ & $\begin{array}{l}\text { Extended-release } \\
\text { tacrolimus }(\boldsymbol{N}=\mathbf{1 9})\end{array}$ & $\begin{array}{l}\text { Immediate-release } \\
\text { tacrolimus }(\boldsymbol{N}=\mathbf{5 5})\end{array}$ & $\boldsymbol{p}$ value \\
\hline Tacrolimus measurements, & 30 & $11.8(1.8)$ & $11.9(2.6)$ & 0.58 \\
mean (SD) & 90 & $25.6(3.6)$ & $26.1(7.1)$ & 0.41 \\
& 365 & $47.0(14.5)$ & $46.9(18.1)$ & 0.51 \\
\hline
\end{tabular}

$S D$ standard deviation

Table 3 Tacrolimus trough levels beyond 30 days post-transplant

\begin{tabular}{llll}
\hline & $\begin{array}{l}\text { Extended-release tacrolimus } \\
(\boldsymbol{N}=\mathbf{1 9})\end{array}$ & $\begin{array}{l}\text { Immediate-release tacrolimus } \\
(\boldsymbol{N}=\mathbf{5 5})\end{array}$ & $\boldsymbol{p}$ value \\
\hline $\begin{array}{l}\text { Tacrolimus trough levels, } \mathrm{ng} / \mathrm{mL}, \\
\text { mean (SD) }\end{array}$ & $8.5(1.2)$ & $7.7(1.3)$ & 0.51 \\
$n$ & 19 & 52 & \\
\hline
\end{tabular}

$S D$ standard deviation

with ER-T, but this difference reached only borderline statistical significance $(1.0 \pm 0.3$ versus $1.5 \pm 1.2 ; p=0.05)$. No statistically significant differences were seen between the two treatment groups regarding the number of outpatient clinic visits within 90 days posttransplantation $(p=0.13)$ and the mean number of readmissions at 30,90, and 365 days posttransplantation $(p=0.38, \quad p=0.41, \quad$ and $p=0.52$, respectively) (Table 4 ). The total number of all-cause hospitalization days within 30 and 90 days post-transplantation was also similar in the ER-T group versus the IR-T group $(p=0.42$ and $p=0.77$, respectively) (Table 4$)$. By 365 days post-transplantation, the number of all-cause hospitalization days was numerically greater with IR-T versus ER-T; however, this difference was not statistically significant ( $3.4 \pm 7.3$ versus $1.4 \pm 2.7$ days; $p=0.86$ ).

There were no emergency room visits for patients receiving ER-T compared with $0.3 \pm 0.9$ visits for patients receiving IR-T within 365 days post-transplantation (Table 4). Significantly fewer renal biopsies were performed within 365 days post-transplantation in the ER-T versus the IR-T group $(0.9 \pm 0.3$ versus $1.6 \pm 0.8$ biopsies; $p=0.01$ ) (Table 4 ).

\section{DISCUSSION}

The aim of this 1-year retrospective analysis exploring clinical outcomes and healthcare resource utilization in matched patients who received ER-T versus IR-T-based maintenance immunosuppression was to further characterize the use of ER-T in a real-world setting. To our knowledge, there is a paucity of data describing outcomes for ER-T under alemtuzumab induction. This is the first study to report the use of ER-T following induction with alemtuzumab in a real-world setting. In addition, while numerous studies have confirmed the safety and efficacy of converting stable adult kidney transplant recipients from IR-T to ER-T [20-23], none of these studies has included patients converted within 10 days after transplantation [24].

This is the first study reporting outcomes using a novel approach of early conversion from IR-T to ER-T for de novo kidney transplant recipients. Overall, no patients receiving the extended-release formulation experienced BCAR or graft failure, required retransplantation, or died. Renal function was also similar over 1 year of follow-up in both groups. 
Table 4 Clinic visits and hospitalization post-transplantation

Parameter

Within 30

Within 90

Within 365

Number of all-cause readmissions (days)

Within 30

Within 90

Within 365

Total number of all-cause hospitalizations (days)

Within 30

Within 90

Within 365
Immediate-release tacrolimus $(N=55)$ $p$ value

tacrolimus $(N=19)$

$\begin{array}{ll}1.0 \pm 0.3 & 1.5 \pm 1.2 \\ {[0.0-2.0]} & {[0.0-8.0]} \\ 2.0 \pm 0.7 & 2.5 \pm 1.8 \\ {[0.0-3.0]} & {[1.0-10.0]} \\ 4.8 \pm 1.3 & 4.4 \pm 2.1 \\ {[2.0-8.0]} & {[1.0-11.0]}\end{array}$

$1.0 \pm 0.3$

0.05

0.13

0.72

[1.0-11.0]

$0.3 \pm 0.5$

0.38

[0.0-2.0]

[0.0-2.0]

$0.6 \pm 1.0$

0.41

[0.0-4.0]

[0.0-4.0]

$1.1 \pm 2.1$

0.52

$$
\text { [0.0-11.0] }
$$

$0.7 \pm 2.3$

0.42

[0.0-14.4]

$$
\text { [0.0-5.0] }
$$

$1.8 \pm 4.5$

0.77

[0.0-25.2]

$$
\text { [0.0-5.0] }
$$

$3.4 \pm 7.3$

0.86

[0.0-32.2]

[0.0-10.0]

Total number of all-cause emergency room visits (days)
Within 30
$0.0 \pm 0.0$
$0.1 \pm 0.3$
Within 90
$[0.0-0.0]$
[0.0-1.0]
Within 365
$0.0 \pm 0.0$
$0.2 \pm 0.4$
[0.0-0.0]
[0.0-2.0]
Within 365
Number of renal biopsies within 365 days post-
transplantation
$0.0 \pm 0.0$
$0.3 \pm 0.9$
[0.0-0.0]
[0.0-6.0]
$0.9 \pm 0.3$
$1.6 \pm 0.8$
[0.0-4.0]

0.01

$S D$ standard deviation

Data are mean \pm SD [range] 
In this study, most patients received induction therapy with alemtuzumab $(57.9 \%)$ or basiliximab $(36.8 \%)$. The regimen was associated with good clinical efficacy, which is in line with low rates of BCAR reported in patients treated with alemtuzumab induction therapy followed by IR-T-based maintenance immunosuppression [25].

Although 1-year graft and patient survival rates were high with both tacrolimus formulations, no patients receiving ER-T experienced graft failure compared with $5.5 \%$ of patients receiving IR-T. Similar 1-year graft and patient survival rates with ER-T and IR-T have been reported previously. For example, in a phase III study of de novo kidney transplant patients, 1-year graft survival rates were $91.5 \%$ and $92.8 \%$, with patient survival rates of $96.9 \%$ and $97.5 \%$ for ER-T and IR-T, respectively [2]. Another phase III study showed that 1-year graft survival rates were $96.7 \%$ and $92.9 \%$, while patient survival rates were $98.6 \%$ and $95.7 \%$ for ER-T and IR-T, respectively [26].

In line with the high graft survival rate, the proportion of patients experiencing a BCAR event was low in both treatment groups and consistent with previous publications. For example, results from a phase II study extension showed a high (93.3\%) BCAR-free survival rate in de novo kidney transplant patients receiving ER-T at 1 year [27], which remained greater than $90 \%$ at 4 years post-transplantation [23]. Furthermore, our study supports the similar rate of BCAR between the extended- and immediaterelease formulations, as reported by Silva et al. (10.3\% versus $7.5 \%$, respectively) [26]. Indeed, it is encouraging that no patients receiving ER-T in our study experienced BCAR, and outcomes appear to be similar in a real-world setting under alemtuzumab induction and an early conversion strategy from IR-T to ER-T.

Importantly, ER-T and IR-T both supported long-term renal function, as evidenced by comparable eGFR levels between formulations over 1 year of follow-up. These observations agree with Silva et al. who reported a comparable mean eGFR with ER-T and IR-T at 1-year post-transplantation $\quad\left(58.6 \mathrm{~mL} / \mathrm{min} / 1.73 \mathrm{~m}^{2}\right.$ versus $59.7 \mathrm{~mL} / \mathrm{min} / 1.73 \mathrm{~m}^{2}$, respectively) [26]. Our findings are also consistent with the 1-year results of a long-term follow-up study by Van Hooff et al., in which mean serum creatinine and creatinine clearance rates remained stable over 4 years in de novo kidney transplant patients receiving ER-T [23]. Likewise, good long-term renal function has been reported in kidney transplant recipients converted from IR-T to ER-T. For example, in the R-EVOLUTION study-a 3-year follow-up of 1798 patients who were receiving ER-T from the EVOLUTION trial $[22,28]$ - the mean eGFR by the Modification of Diet in Renal Disease- 4 remained stable over 3 years after conversion from IR-T $(56.7 \mathrm{~mL} /$ $\mathrm{min} / 1.73 \mathrm{~m}^{2}$ versus $58.1 \mathrm{~mL} / \mathrm{min} / 1.73 \mathrm{~m}^{2}$ at baseline and year 3 , respectively) [28].

In this study, the time taken to achieve target tacrolimus trough levels was not significantly different between ER-T and IR-T (9.2 versus 7.8 days). Both the number of dose adjustments required to attain target levels and the tacrolimus trough levels on or after 30 days of treatment were also similar between both formulations. Furthermore, these data are consistent with a study of de novo kidney transplant patients in which systemic tacrolimus exposure was similar for both formulations at steady state (following dose adjustment), as evidenced by a comparable area under the concentration-time curve at day 14 and at week 6 [27]. These data suggest that initiating ER-T in a real-world setting is no more complex than it is with IR-T, and that early conversion from IR-T to ER-T post-transplant yields similar outcomes to that of traditional, continuous treatment with IR-T. Importantly, ER-T provides patients with the option of once-daily dosing of tacrolimus.

Given the reported positive clinical outcomes with ER-T-based immunosuppression, several studies have assessed the economic impact of treating kidney transplant patients with this formulation versus IR-T [29-31]. Such studies have predicted that ER-T would be associated with a range of cost savings due to improved rejection and graft survival rates [29-31]. For example, using cost data from Medicare and the US Renal Data System, Abecassis et al. showed that ER-T was associated with a 5-year cost saving of US\$9411 [31]. This was driven largely by a reduction in the costs 
associated with graft loss and dialysis compared with IR-T [31]. While our study did not directly assess cost savings, we did not find statistically significant differences between treatment groups for the number of outpatient clinic visits, readmissions, hospitalization days, and emergency room visits. However, nonsignificant differences in outpatient clinic visits during the first 90 days, and total hospitalization days during the first 365 days, may have reached statistical significance with a larger number of patients.

A number of limitations in the design of this study should be noted. As this was a retrospective study, a number of study biases are likely to be inherent. First, the number of patients who were available for this study based on the inclusion criteria was small. The study was also undertaken shortly after the introduction of ER-T into the US market when a paucity of realworld data were available characterizing the use of ER-T. The small sample size may have affected the power to detect differences between the two treatment groups. In addition, the centerspecific practice of capping the measured eGFR values at $60 \mathrm{~mL} / \mathrm{min} / 1.73 \mathrm{~m}^{2}$ may have limited the ability to fully assess differences in renal function over time between the two groups. As this study explored the impact of ER-T versus IR-T on clinical and healthcare resource utilization parameters over 1 year only, longerterm differences between the formulations could not be detected. This is noteworthy in that nonadherence to treatment, which has a substantial impact on antibody-mediated rejection, graft survival, and costs [11, 13, 32, 33], is known to increase over time [34].

There are several strengths of this study that should be noted. For instance, propensity score matching against a number of characteristics helped control for bias in patient selection between treatment groups. Furthermore, we used a large patient database, which provided real-world data outside of the controlled environment of an RCT. As such, we anticipate that our findings may be more generalizable to other transplant populations receiving ER-T or IR-T in routine clinical settings than data generated from RCTs.

\section{CONCLUSION}

While ER-T has been available in Europe for almost a decade, to our knowledge this is the first study examining clinical outcomes and healthcare resource utilization of kidney transplant patients treated with the extended-release formulation under US real-world practice conditions. Exploring a real-world data set also enabled us to characterize a unique treatment approach: early conversion from IR-T to ER-T under alemtuzumab induction in de novo kidney transplant recipients. As such, this study affirms that ER-T can be reliably employed in this practice setting, while achieving promising outcomes on a par with those seen in several large European trials. Healthcare resource utilization was generally similar between treatments; however, there were fewer outpatient visits with ER-T at borderline statistical significance $(p=0.05)$. Further investigation in larger real-world studies is warranted to fully characterize the real-world clinical, economic, and resource utilization outcomes with the extended- versus immediate-release formulation employing the use of novel treatment approaches not captured in controlled clinical trials.

\section{ACKNOWLEDGEMENTS}

Assistance with chart reviews was provided by Clinical Research Core, Comprehensive Transplant Center, Northwestern University Feinberg School of Medicine.

Funding. This study, the journal's article processing charges, and open access fee were funded by Astellas Pharma Global Development, Inc. All authors had full access to all of the data in this study and take complete responsibility for the integrity of the data and the accuracy of the data analysis.

Medical Writing and Editorial Assistance. James Wallis, MRes, from Cello Health MedErgy assisted in drafting the initial version of the manuscript under the direction of the authors, and provided editorial support 
throughout its development. Editorial support was funded by Astellas Pharma, Inc.

Authorship. All named authors meet the International Committee of Medical Journal Editors (ICMJE) criteria for authorship for this article, take responsibility for the integrity of the work as a whole, and have given their approval for this version to be published.

Authorship Contributions. All authors contributed to drafting the article and data analysis. BH, SEM, LZ, AIS, and DPL contributed to research design and performance of the research. HB, EL, RK, and JJS contributed to research design. KA contributed to performance of the research.

Disclosures. Bing Ho reports a contract with Astellas to conduct the study research. Hardik Bhagat is employed by Astellas Pharma US, Inc. and has received a salary from Astellas Pharma US, Inc. Edward Lee is a former employee of Astellas, and has received a salary from Astellas. Kofi Atiemo reports a contract with Astellas to conduct the study research. Amna Daud reports a contract with Astellas to conduct the study research. Raymond Kang reports a contract with Astellas to conduct the study research. Samantha E. Montag reports a contract with Astellas to conduct the study research. Lihui Zhao reports a contract with Astellas to conduct the study research. Jason J. Schwartz is employed by Astellas and has received a salary from Astellas. Anton I. Skaro reports a contract with Astellas to conduct the study research. Daniela P. Ladner reports a contract with Astellas to conduct the study research.

Compliance with Ethics Guidelines. This retrospective database analysis study had approval of the Northwestern University Institutional Review Board (IRB\#STU0020221) and adhered to the 1964 Helsinki declaration and its later amendments or comparable ethical standards. As a retrospective study, informed consent of individuals was not required.

Data Availability. Access to anonymized individual patient-level data will not be provided for this study as it meets one or more of the exceptions described under the Sponsor Specific Information for Astellas on http://www. clinicalstudydatarequest.com.

Open Access. This article is distributed under the terms of the Creative Commons Attribution-NonCommercial 4.0 International License (http://creativecommons.org/licenses/ by-nc/4.0/), which permits any noncommercial use, distribution, and reproduction in any medium, provided you give appropriate credit to the original author(s) and the source, provide a link to the Creative Commons license, and indicate if changes were made.

\section{REFERENCES}

1. Silva HT Jr, Yang HC, Meier-Kriesche HU, et al. Long-term follow-up of a phase III clinical trial comparing tacrolimus extended-release/MMF, tacrolimus/MMF, and cyclosporine/MMF in de novo kidney transplant recipients. Transplantation. 2014;97:636-41.

2. Krämer BK, Charpentier B, Bäckman L, et al. Tacrolimus once daily (ADVAGRAF) versus twice daily (PROGRAF) in de novo renal transplantation: a randomized phase III study. Am J Transplant. 2010;10:2362-643.

3. Stifft F, Stolk LM, Undre N, van Hooff JP, Christiaans $\mathrm{MH}$. Lower variability in 24-hour exposure during once-daily compared to twice-daily tacrolimus formulation in kidney transplantation. Transplantation. 2014;97:775-80.

4. Wu MJ, Cheng CY, Chen $\mathrm{CH}$, et al. Lower variability of tacrolimus trough concentration after conversion from Prograf to Advagraf in stable kidney transplant recipients. Transplantation. 2011;92:648-52.

5. Kuypers DR, Peeters PC, Sennesael JJ, et al. Improved adherence to tacrolimus once-daily formulation in renal recipients: a randomized controlled trial using electronic monitoring. Transplantation. 2013;95:333-40.

6. Eberlin M, Otto G, Krämer I. Increased medication compliance of liver transplant patients switched from a twice-daily to a once-daily tacrolimus-based immunosuppressive regimen. Transplant Proc. 2013;45:2314-20. 
7. Beckebaum S, Iacob S, Sweid D, et al. Efficacy, safety, and immunosuppressant adherence in stable liver transplant patients converted from a twice-daily tacrolimus-based regimen to once-daily tacrolimus extended-release formulation. Transpl Int. 2011;24:666-75.

8. Sapir-Pichhadze R, Wang Y, Famure O, Li Y, Kim SJ. Time-dependent variability in tacrolimus trough blood levels is a risk factor for late kidney transplant failure. Kidney Int. 2014;85:1404-11.

9. Pollock-BarZiv SMS, Finkelstein Y, Manlhiot C, et al. Variability in tacrolimus blood levels increases the risk of late rejection and graft loss after solid organ transplantation in older children. Pediatr Transplant. 2010;14:968-75.

10. Wiebe C, Gibson IW, Blydt-Hansen TD, et al. Evolution and clinical pathologic correlations of de novo donor-specific HLA antibody post kidney transplant. Am J Transplant. 2012;12:1157-67.

11. Wiebe C, Gibson IW, Blydt-Hansen TD, et al. Rates and determinants of progression to graft failure in kidney allograft recipients with de novo donorspecific antibody. Am J Transplant. 2015;15:2921-30.

12. Rodrigo E, Segundo DS, Fernández-Fresnedo G, et al. Within-patient variability in tacrolimus blood levels predicts kidney graft loss and donor-specific antibody development. Transplantation. 2016;100:2479-85.

13. Sellarés J, de Freitas DG, Mengel M, et al. Understanding the causes of kidney transplant failure: the dominant role of antibody-mediated rejection and nonadherence. Am J Transplant. 2012;12:388-99.

14. Hart A, Smith JM, Skeans MA, et al. OPTN/SRTR 2015 annual data report: kidney. Am J Transplant. 2017;17(Suppl 1):21-116.

15. Gondos A, Döhler B, Brenner H, Opelz G. Kidney graft survival in Europe and the United States: strikingly different long-term outcomes. Transplant J. 2013;95:267-74.

16. Merion RM, Goodrich NP, Johnson RJ, et al. Kidney transplant graft outcomes in 379257 recipients on 3 continents. Am J Transplant. 2018;18:1914-23.

17. Tanzi MG, Undre N, Keirns J, Fitzsimmons WE, Brown M, First MR. Pharmacokinetics of prolongedrelease tacrolimus and implications for use in solid organ transplant recipients. Clin Transplant. 2016;30:901-11.

18. Einecke G, Sis B, Reeve J, et al. Antibody-mediated microcirculation injury is the major cause of late kidney transplant failure. Am J Transplant. 2009;9:2520-31.

19. National Institute of Diabetes and Digestive and Kidney Diseases (NIDDK). Estimating glomerular filtration rate (GFR). NIDDK. 2017. https://www. niddk.nih.gov/health-information/communicationprograms/nkdep/laboratory-evaluation/glomerularfiltration-rate/estimating. Accessed 14 Mar 2019.

20. Alloway R, Steinberg S, Khalil K, et al. Conversion of stable kidney transplant recipients from a twice daily Prograf-based regimen to a once daily modified release tacrolimus-based regimen. Transplant Proc. 2005;37:867-70.

21. Van Hooff J, Van der Walt I, Kallmeyer J, et al. Pharmacokinetics in stable kidney transplant recipients after conversion from twice-daily to once-daily tacrolimus formulations. Ther Drug Monit. 2012;34:46-52.

22. Guirado L, Cantarell C, Franco A, et al. Efficacy and safety of conversion from twice-daily to once-daily tacrolimus in a large cohort of stable kidney transplant recipients. Am J Transplant. 2011;11:1965-71.

23. van Hooff JP, Alloway RR, Trunečka P, Mourad M. Four-year experience with tacrolimus once-daily prolonged release in patients from phase II conversion and de novo kidney, liver, and heart studies. Clin Transplant. 2011;25:1-12.

24. Caillard S, Moulin B, Buron F, et al. Advagraf ${ }^{\circledR}$, a once-daily prolonged release tacrolimus formulation, in kidney transplantation: literature review and guidelines from a panel of experts. Transpl Int. 2016;29:860-9.

25. Hanaway MJ, Woodle ES, Mulgaonkar S, et al. Alemtuzumab induction in renal transplantation. N Engl J Med. 2011;364:1909-19.

26. Silva HT Jr, Yang HC, Abouljoud M, et al. Erratum: one-year results with extended-release tacrolimus/ $\mathrm{MMF}$, tacrolimus/MMF and cyclosporine/MMF in de novo kidney transplant recipients. Am J Transplant. 2007;7:595-608.

27. Wlodarczyk Z, Squifflet JP, Ostrowski M, et al. Pharmacokinetics for once- versus twice-daily tacrolimus formulations in de novo kidney transplantation: a randomized, open-label trial. Am J Transplant. 2009;9:2505-13.

28. Guirado L, Burgos D, Cantarell C, et al. Mediumterm renal function in a large cohort of stable kidney transplant recipients converted from twicedaily to once-daily tacrolimus. Transplant Direct. 2015;1:e24. 
29. Muduma G, Odeyemi I, Pollock RF. Evaluating the economic implications of non-adherence and antibody-mediated rejection in renal transplant recipients: the role of once-daily tacrolimus in the UK. J Med Econ. 2015;18:1050-9.

30. Muduma G, Odeyemi I, Smith-Palmer J, Pollock RF. Budget impact of switching from an immediate-release to a prolonged-release formulation of tacrolimus in renal transplant recipients in the UK based on differences in adherence. Patient Prefer Adherence. 2014;8:391-9.

31. Abecassis MM, Seifeldin R, Riordan ME. Patient outcomes and economics of once-daily tacrolimus in renal transplant patients: results of a modeling analysis. Transplant Proc. 2008;40:1443-5.
32. Gaynor JJ, Ciancio G, Guerra G, et al. Graft failure due to noncompliance among 628 kidney transplant recipients with long-term follow-up. Transplantation. 2014;97:925-33.

33. Pinsky BW, Takemoto SK, Lentine KL, Burroughs TE, Schnitzler MA, Salvalaggio PR. Transplant outcomes and economic costs associated with patient noncompliance to immunosuppression. Am J Transplant. 2009;9:2597-606.

34. Ichimaru N, Kakuta $\mathrm{Y}$, Abe $\mathrm{T}$, et al. Treatment adherence in renal transplant recipients: a questionnaire survey on immunosuppressants. Transplant Proc. 2008;40:1362-5. 